\title{
EMBARAZO EN MUJERES EN SITUACIÓN DE PROSTITUCIÓN: AUTOCUIDADO Y PRÁCTICAS DE RIESGO. ESTUDIO CUALITATIVO. BOGOTÁ, COLOMBIA 2006
}

\author{
A qualitative study of pregnancy in women working \\ in prostitution in Bogotá, Colombia during 2006: \\ self-care and high risk practices \\ María Mercedes Lafaurie*, Pío Iván Gómez-Sánchez, M.D.***, \\ Andrea Juliana Bernal***, María Angélica Gualteros***, \\ Viviana Patricia Hassan***, Jenny Amparo Lozano***, Claudia Viviana Tovar*** \\ Recibido: mayo 9/08 - Aceptado: noviembre 21/08
}

\section{RESUMEN}

Objetivos: identificar las prácticas de autocuidado de mujeres embarazadas en situación de prostitución, de la zona de alto impacto de las localidades Santa Fe y los Mártires, de Bogotá D.C., así como las condiciones en que se presenta el embarazo y las posibles prácticas de riesgo.

Métodos: del 24 de julio al 31 de octubre de 2006, se realizó una aproximación cualitativa, con perspectiva de género, a las condiciones en las que viven sus embarazos, riesgos maternos y comportamientos de autocuidado, un grupo de 12 mujeres en situación de prostitución de las zonas de alto impacto de las localidades Santa Fe y los Mártires, de Bogotá D.C. Se obtuvo la información mediante entrevistas a profundidad, grabadas y posteriormente transcritas, previa aprobación verbal y escrita. El análisis de la información fue realizado con el apoyo del software Atlas Ti 5.0.

Resultados: se encontraron riesgos asociados a sexo inseguro y embarazo no deseado; las prácticas

* Profesora Asociada, Universidad El Bosque. Bogotá, Colombia.

** Profesor Titular, Universidad Nacional de Colombia. Bogotá, Colombia. Correo electrónico: gesypro_fmbog@unal.edu.co

*** Enfermeras Universidad El Bosque. Bogotá, Colombia. de autocuidado se reducen al uso del condón con los clientes, más no con sus parejas. Las mujeres reportan disminución de la actividad sexual, cambio en algunas prácticas sexuales y disminución del consumo de alcohol, tabaco y substancias psicoactivas. Siete de las 12 mujeres no usaban anticoncepción; dos atribuyen su embarazo a fallas del condón.

Conclusiones: más que considerar su actividad un riesgo para su embarazo, su embarazo "pone en riesgo" su actividad. En este grupo se encuentran varios factores de riesgo que amenazan la salud materna, por ello se recomienda la educación enfocada en anticoncepción, autocuidado y empoderamiento.

Palabras clave: prostitución, factores de riesgo, autocuidado, infecciones de transmisión sexual, anticoncepción, género.

\section{SUMMARY}

Objectives: this research was aimed at learning about self-care practices in pregnant women in prostitution in the high impact Mártires and Santa Fe neighbourhoods of Bogotá, as well as the conditions of their current pregnancies and possible practices putting them at risk. 
Methods: a qualitative gender-based approach was adopted with 12 women who were recruited from July 24th to October 31st 2006 to ascertain conditions regarding their pregnancy, risks factors and self-care practices. The information was gathered by in-depth interviews following the women's verbal and written approval; such interviews were recorded and later transcribed. Atlas Ti 5.0 software was used for analysing the data.

Results: risks associated with unsafe sex and unwanted pregnancies were identified. Condom use with clients was the only self-care practice found; such practice did not extend to being used with their couples. The women reported decreased sexual activity and changes in some sexual practices. They also reported decreased alcohol, tobacco and psychoactive substance use. Seven of the twelve women did not use contraception. Two women attributed their pregnancies to condom failure.

Conclusions: instead of considering their activity as a risk for their pregnancies, the women regarded becoming pregnant as putting their work at risk. We found many risk factors for maternal health in this group which is why we recommend focusing their education on issues such as contraception, self-care practices and female empowerment.

Key words: prostitution, risk factor, self-care, sexually-transmitted infection, contraception, gender.

\section{INTRODUCCIÓN}

La pobreza, desnutrición y violencia son aspectos que afectan la calidad de vida de las mujeres en estado de embarazo. ${ }^{1}$ Un caso especial son la mujeres que ejercen la prostitución, quienes presentan problemas como el consumo de alcohol, el riesgo de contraer ITS/VIH/SIDA y la violencia callejera, lo cual las coloca en alta vulnerabilidad. ${ }^{2,3}$ Lo anterior representa condiciones de sometimiento en las cuales ejercen su actividad y que conllevan a múltiples riesgos que potencialmente pueden afectar su salud y la del producto de la gestación.

Llevar a cabo aproximaciones hacia la situación de salud de estas mujeres, especialmente cuando se encuentran en estado de embarazo, ayuda a contar con elementos, desde la perspectiva del profesional de la salud, para reducir los riesgos y mejorar su calidad de vida. Los profesionales de la salud son quienes más fácilmente pueden detectar los factores de riesgo asociados a prácticas sexuales, utilización incorrecta de métodos anticonceptivos, consumo de alcohol y sustancias psicoactivas (SPA) durante la gestación, y los hábitos de higiene genital, entre otros, que conllevan al deterioro precoz de la salud de la mujer y de la población infantil.., Es importante conocer, desde un abordaje integral, el contexto en el que se desenvuelven las personas en situación de prostitución, la manera como evitan el riesgo, las redes de apoyo social con que cuentan y la conciencia de autocuidado que tienen con respecto a las ITS durante el embarazo. Parte del abordaje integral incluye la perspectiva de género.

Es escasa la información disponible acerca de esta problemática en la literatura científica disponible, tanto en Colombia como en Latinoamérica. Se encuentran numerosos estudios relacionados con la prevención y promoción de ITS/VIH/SIDA en mujeres en situación de prostitución, ${ }^{6}$ así como con la prevención de embarazos no deseados, pero no en asociación con el embarazo. ${ }^{7,8}$ De otra parte, se han realizado múltiples procesos de educación para la prevención de ITS/VIH/SIDA con mujeres inmersas en esta cultura, pero el embarazo visto desde una perspectiva integral, así como la maternidad, son aspectos que muy poco han sido abordados en relación con este grupo humano.

La Subdirección de Integración Social de los Mártires, la cual tiene una larga trayectoria en el trabajo con la población en situación de prostitución de la zona de los Mártires y Santa Fe, permitió al equipo de investigación acceder a mujeres en situación de prostitución que asisten al programa de capacitación destinado a ellas, y a partir de este contacto ubicar posibles participantes en este estudio.

Teniendo en cuenta lo anterior, se planteó el presente estudio con el propósito de conocer las prácticas de autocuidado en mujeres embarazadas 
en situación de prostitución, las circunstancias en las cuales se vivencian sus embarazos, el significado que tiene para ellas su estado, las prácticas de riesgo propias de este grupo de mujeres, las redes sociales de apoyo con las que cuentan y los posibles riesgos maternos. Esta información permite contar con referentes para encaminar posteriores procesos preventivos y de apoyo social.

\section{MÉTODOS}

La presente investigación se desarrolló con metodología cualitativa, realizando entrevistas a profundidad a mujeres embarazadas en situación de prostitución, indagando sobre aspectos sociales y prácticas culturales, y el lugar en que desempeñan su trabajo, teniendo en cuenta la perspectiva de las participantes.

\section{Consideraciones éticas}

De acuerdo con la resolución $\mathrm{N}^{\circ} 008430$ de 1993, y debido a que se tocaron aspectos sensibles del comportamiento, esta investigación se considera de bajo riesgo. El consentimiento informado utilizado fue de tipo verbal, con aceptación escrita por parte de cada una de las participantes; las entrevistas fueron grabadas con autorización de cada una de ellas. Para preservar la privacidad de las entrevistadas, se utilizaron nombres ficticios.

Se entrevistaron, del 24 de julio al 31 de octubre de 2006, doce mujeres en estado de embarazo, que ejercen la prostitución en la zona de alto impacto de las localidades los Mártires y Santa Fe, en el centro de la ciudad de Bogotá, sector donde priman los negocios relacionadas con la venta de alcohol, el comercio sexual y los lugares de diversión para adultos. Se excluyeron del estudio las mujeres que no desearon ser entrevistadas.

\section{Procedimiento}

Se estableció contacto con la Subsecretaría Distrital de Integración de los Mártires, la cual desarrolla actividades de capacitación en desarrollo personal, salud sexual y reproductiva y derechos humanos con población en prostitución. A través de talleres en salud sexual y reproductiva, en los cuales el equipo de investigación prestó su apoyo, se establecieron contactos con algunas mujeres en situación de embarazo. Haciendo uso de la técnica de "bola de nieve" se logró finalmente captar 12 mujeres, cuya información permitió observar tendencias y caracterizar el grupo y su contexto. Se llevaron a cabo entrevistas a profundidad, con el apoyo de una guía construida para tal fin, con duración de una y media a dos horas. Las entrevistas fueron realizadas por dos personas del equipo de investigación, una de las cuales tuvo como misión desarrollar la entrevista, la cual fue grabada para facilitar su transcripción, mientras la otra tomó notas y observó el lenguaje no verbal de la entrevistada.

\section{Variables a observar}

Caracterización socio-demográfica, situación familiar (convivencia o no en pareja, número de hijos), caracterización de la actividad de prostitución (en cuanto a tiempo de dedicación, lugar donde se realiza, modo de captación del cliente, horarios, número de clientes por día, prácticas sexuales, ingresos, lugar de residencia), antecedentes familiares (familia de origen, vida intrafamiliar y relaciones actuales con ésta), estado de salud (antecedentes traumáticos, patológicos, toxico-alérgicos, ITS, violencia sexual), historia sexual y reproductiva (tiempo de gestación, número de embarazos, número de abortos, edad de iniciación de la vida sexual, anticoncepción, circunstancias del embarazo actual, percepción de la maternidad), cambios en el ejercicio de la actividad debidos al embarazo, autocuidado y autoprotección (uso de protección sexual, asistencia a controles y curso de preparación, alimentación), proyecto de vida y experiencia con los servicios de salud.

\section{Análisis}

Una vez obtenida la información, ésta fue transcrita y procesada mediante el software alemán Atlas Ti 5, destinado a la codificación de información cualitativa. ${ }^{9}$ Posteriormente, se realizaron cuadros descriptivos y se llevó a cabo la interpretación de los 
hallazgos a la luz del marco de referencia y el marco teórico. Una vez realizado el análisis, se procedió a llevar a cabo la discusión de los resultados y a plantear conclusiones y recomendaciones.

El proceso de establecimiento de categorías de análisis, fue realizado por parte del equipo de investigación, con base en el marco teórico. Se realizó la triangulación de los resultados mediante el método de acuerdo entre investigadores independientes.

\section{RESULTADOS}

Las edades de las mujeres entrevistadas están entre los 16 y los 42 años, una de ellas, por ser menor de edad, es considerada víctima de explotación sexual. Once de las doce participantes provienen de zonas urbanas y han accedido a la educación secundaria. Los lugares de procedencia de estas mujeres son los siguientes: Bogotá (3 mujeres); Montería, Córdoba (2 mujeres); Cúcuta, Santander del Norte (1 mujer); Medellín, Antioquia (1 mujer); Pereira, Risaralda (1 mujer); San José del Guaviare, Guaviare (1 mu- jer); Neiva, Huila (1 mujer); Cali, Valle (1 mujer), e Isla Fuerte, Bolívar (1 mujer). Tres mujeres están afiliadas al régimen contributivo de salud, otras tres al régimen subsidiado, tres son atendidas como vinculadas y otras tres no han accedido a servicios de salud.

En la tabla 1 se caracteriza la actividad según el lugar donde se realiza, tiempo diario dedicado a la misma, tiempo que llevan las mujeres en situación de prostitución, ingresos mensuales, número de clientes al día, lugar de residencia y horario de la actividad.

En 9 casos esta actividad se desarrolla en establecimientos donde se presta servicio de bar a los clientes, mientras que en 3 de ellos en la calle. Entre los establecimientos mencionan sobre todo los reservados, los bares y las "whiskerías”; quienes realizan trabajo de calle suelen ir con sus clientes a residencias. El hacer ingerir alcohol a los clientes hace parte importante de la actividad de prostitución, por lo tanto las mujeres suelen ganar también

\begin{tabular}{|l|c|c|}
\hline \multicolumn{2}{|c|}{ Tabla 1. Caracterización de la actividad. } \\
\hline Concepto & Valores & Casos \\
\hline Lugar donde realiza la actividad & Calle & 3 \\
\hline & Bar o establecimiento & 9 \\
\hline Tiempo diario dedicado a la actividad & Menos de 4 horas & 1 \\
\hline & $4-8$ horas & 5 \\
\hline Tiempo en situación de prostitución & Más de 8 horas & 6 \\
\hline & Menos de 1 año & 4 \\
\hline Ingresos mensuales & 1 a 5 años & 7 \\
\hline & 6 o más años & 0 \\
\hline Número de clientes por día & Sin dato & 1 \\
\hline ¿Habita en el lugar donde realiza la actividad? & Menos del mínimo & 0 \\
\hline & Mínimo & 1 \\
\hline & Más del mínimo & 11 \\
\hline & Menos de 2 & 1 \\
\hline
\end{tabular}


un porcentaje sobre estas ventas. Esto hace que sea muy frecuente en ellas este consumo. La actividad que ellas denominan "mesear" es aquella en que las mujeres suscitan el consumo de alcohol en los clientes, sentándose con ellos a departir en su mesa.

En este momento el precio por lo que ellas denominan "el rato" con el cliente gira alrededor de los \$35.000; si realizan actividades, como las denominadas "presentaciones" o show, pueden obtener entre $\$ 15.000$ y $\$ 20.000$.

Las razones económicas son aquellas que argumentan con mayor frecuencia para justificar su dedicación a la prostitución. Con respecto a las prácticas sexuales que llevan a cabo, la gran mayoría menciona que hacen "lo normal", es decir coito vaginal, sexo oral y lo que las mujeres llaman "poses" o actividades de exhibicionismo. El coito anal suele ser rechazado por ellas.

\section{Vida familiar}

Solamente dos de las mujeres viven en pareja y mencionan que su relación es estable. Cinco de las mujeres tienen relaciones esporádicas con su pareja, quienes en su gran mayoría son los padres de los hijos(as) que esperan y en varias ocasiones también de sus restantes. Se trata a menudo de relaciones en las que son ellas quienes corren con las responsabilidades económicas. Paola, de 25 años de edad, comenta lo siguiente acerca de su pareja:

“'No ve que él está preso y a mí me toca darle a él? pues la visita, cuando voy a visitarlo lo normal, los útiles de aseo, las cosas que él necesite"

La inestabilidad del vínculo de pareja se refleja así en el caso de Julieta: "En estos momentos sola, pues o sea por ratos el papá de mis hijos... por ahí estuvo y nos vemos, así... pero no estoy viviendo estable con él, eso es así unos dítas y él luego se va otra vez y así..."

Cinco mujeres relatan que se encuentran solas, sin pareja. Así lo expresa, por ejemplo, Marilyn, de 23 años: "Nadie me jode la vida"

Diez de las doce participantes ya tienen hijos, y éstos en la mitad de los casos son educados por personas diferentes a ellas, la mayoría miembros de su familia. En 4 casos en los cuales ellas conservan consigo sus hijos, éstos permanecen con amigas, vecinas o familiares.

En 7 casos las mujeres tienen contacto con sus familias de origen, mientras en 5, las relaciones con éstas son ausentes. En algunas ocasiones se trata de casos en los cuales las mujeres han migrado recientemente de sus lugares de origen.

En otros casos, las mujeres que con frecuencia provienen de familias desintegradas, abandonaron tempranamente su hogar por razones de maltrato. Relatos como el de Sara son frecuentes entre las mujeres de este grupo, sobre todo entre aquellas que llevan varios años dedicadas a la actividad: "Pues de mi papá, uhm... lo alcancé a conocer como a los diecisiete años, no volví a saber nada de él, de mi mamá pues nunca nos llevamos bien, siempre me pegaba, siempre me maltrataba, pero no, ni idea porque siempre estuvimos muy alejadas desde que tuve conocimiento y desde que conocí mi primera pareja me fui de la casa"

\section{Estado de salud}

Dos del total de las participantes presentan antecedentes alérgicos. En 5 casos han presentado intoxicaciones por diferentes sustancias, como alcohol adulterado, alimentos o veneno para ratas, que han puesto en riesgo su vida. De igual manera, en otros 5 casos las participantes tienen antecedentes patológicos (1 sinusitis, 1 amenaza de aborto, 1 dengue y 1 sífilis), mientras que 7 no reportan enfermedades que hayan afectado seriamente su salud. En la mitad de los casos (6 mujeres) reportaron que no han presentado antecedentes traumáticos, en cambio en la cuarta parte de los casos las mujeres afirman que sí los han presentado por razones como atracos, fracturas y caídas; 3 de ellas no informaron al respecto.

En la mitad de los casos las entrevistadas afirman que presentan infecciones de transmisión sexual (además del caso de sífilis, 5 reportan vaginitis). Jenny relata que estando en el ejercicio de su actividad, el condón que estaba usando su cliente se "zafó" del pene, quedando en el interior de su 
vagina. Por esta razón, según cuenta ella, además de haber quedado en situación de embarazo, contrajo sífilis.

Es importante destacar que 4 de las 12 participantes refieren antecedentes de violencia sexual intrafamiliar en la preadolescencia, a pesar de que la violencia física y psicológica es un antecedente que poco resaltan las mujeres de este grupo dentro de sus historias de vida, siendo algo que consideran prácticamente obvio en su condición de mujeres. No obstante, al indagar a profundidad acerca de los antecedentes de salud, se observa que los golpes y el maltrato por parte de sus parejas son frecuentes en la cotidianidad de las participantes. Laura de 42 años, quien tuvo dos abortos como producto de la violencia física y psicológica de su pareja relató: "Fue que él me pegó, y.... se me vino; me pegó en la parte de las caderas con la peinilla, o sea, un machete. El segundo fue provocado, pues como él no quería que yo tuviera más niños en ese tiempo, por lo que la niña estaba todavía muy pequeñita, entonces él me llevo al consultorio y allá me lo hicieron. Eso fue como en la Caracas, pero yo no me acuerdo con qué, pero eso fue en un consultorio privado. Me pesó, toda la vida me ha pesado..."

\section{Hábitos perjudiciales para la salud}

La tercera parte de las entrevistadas consume tabaco; ocho de las doce afirman consumir alcohol, hecho que hace parte de la cultura en la cual se hallan inmersas. En cuanto al consumo de SPA, la cuarta parte de las entrevistadas inhalan "perico" (alcaloide que se obtiene de las hojas de la planta de la coca), "bóxer" (nombre comercial de pegante industrial, que inhalado es estimulante y suprime el apetito) y/o fuman marihuana. Una de ellas hace uso de tranquilizantes. Varias manifiestan haber disminuido el consumo de alcohol y de SPA a partir del momento en que supieron de su estado de embarazo.

\section{Situación actual de salud}

La percepción de su situación de salud de dos terceras partes del grupo de mujeres es buena, de acuerdo con los datos reportados por ellas. Cuatro mujeres consideran que su estado de salud es complicado, por sus antecedentes patológicos.

\section{Historia sexual - reproductiva}

En la tabla 2 se resumen aspectos que permiten observar de manera integral la historia sexual y reproductiva de este grupo.

Laura ha tenido 6 embarazos a sus 42 años y dos experiencias de aborto, que relata fueron producto de violencia por parte de su pareja, lo cual constituye otro caso de violencia intrafamiliar que hace parte de las historias de vida narradas por las participantes en este estudio.

Paulina narra, por su parte, una historia de aborto fallido. Su embarazo en el momento de la entrevista había seguido su curso, causándole mucha preocupación su situación hacia el futuro: "Algunas de mis compañeras me aconsejaron que fuera a una droguería; me tomé unas pastas, yo sabía que no estaba bien, que eso es quitarle la vida a alguien, sino que yo tengo tres criaturas y yo sé que eso me iba pues a parar todos mis planes, las cosas que yo tenia, imagínese todo iba a quedar estancado porque voy a depender de otra persona y ahora otra criatura, más responsabilidades, no, entonces qué te iba a decir, bueno sin embargo (llorando) mejor dicho de lo peor como se dice, me fui me hice aplicar una inyección, me tomé cuatro pastillas de "Cytotec" (nombre comercial del misoprostol), y después una inyección "ratapoit" creo que era que se llamaba, eso me dijo el señor, me aplicaron eso, me cobraron 100.000 pesos y eso no hizo ningún efecto en mí”

La mitad de las mujeres se encontraban al momento de la entrevista en el primer trimestre de gestación, y la otra mitad entre el segundo y tercer trimestre.

El embarazo en ninguno de los casos fue planeado y constituye para todas las mujeres una situación complicada, bien sea porque sus parejas no han estado de acuerdo, porque consideran tener ya los hijos que desean o porque no desean tener hijos. Quienes están menos afectadas por esta situación son las dos mujeres que conviven con sus parejas, lo 
Tabla 2. Historia sexual-reproductiva.

Concepto

Edad gestacional

Edad inicio vida sexual

Aborto previo

Deseo de futuros embarazos

Embarazo planeado
Valores

Número de casos

Número de embarazos (incluye el actual)

Ed inicio vida sexual

cual corrobora la importancia del vínculo de pareja en la manera como las mujeres de nuestra cultura asumen la maternidad.

Para Paulina la noticia fue desconsoladora: "Yo estuve llorando como veinte días seguidos, mejor dicho yo ni trabajaba, todo el tiempo lo perdí”

Esto manifiesta, por su parte, Marilyn: "No me gustan los chinos"

En 5 de los casos, las mujeres afirman haber estado usando métodos anticonceptivos cuando quedaron embarazadas. Una asegura que estaba utilizando dispositivo intrauterino (DIU); dos aseguran haberse realizado ligadura de trompas, mientras dos afirman que les falló el condón (en un caso se rompió y en el otro se deslizó al interior de la vagina).

Por su parte, María comenta que tenía ligadura de trompas, método que, según ella, falló: "Empecé a vomitar y mal y maluca, maluca, entonces hasta que... hasta que vine y me hice la prueba de embarazo y me salió cierta y entonces pues de ahí, este, ahí sí, pues, me, me, me tocó, o sea, es que el problema era que yo no podía tener bebé porque yo era de las trompas amarradas, pero como ya tiene el niño 7 años, pues pudo ser que se soltaron"

Juliana afirma que ella hace uso del condón con sus clientes; ella, como sus compañeras, no lo utiliza con su pareja.

A pesar de no haber sido planeado su embarazo, la mayoría de las participantes lo aceptan por su deber ser como mujeres y porque la idea de un aborto constituye un peso moral para ellas. Esto comenta Paola: "Para mí, pues ahorita yo no pensaba tener un hijo porque ya con los que tengo basta y hasta me sobra, pero si me llegó pues tengo que tenerlo ino? porque donde yo me ponga a abortarlo me caen, hasta Dios me puede castigar en otras cosas"

Buena parte de ellas (9) no desearían tener futuros embarazos; cuatro afirman que piensan realizarse la ligadura de trompas inmediatamente después del parto. 


\section{Controles durante el embarazo}

Durante el embarazo, una tercera parte del total de las participantes asisten a controles prenatales mensualmente y 4 de ellas relatan no asistir al control prenatal por las siguientes razones: por no contar con documentos, por pereza y por no tener seguridad social. En cuanto a la asistencia al curso de preparación para la maternidad y paternidad responsable, se observa que 6 de las mujeres entrevistadas no asisten, argumentando que no lo consideran importante, no cuentan con tiempo o se preparan ellas mismas.

\section{Hábitos alimenticios}

Únicamente la mitad de este grupo de mujeres consume tres comidas diarias, la tercera parte de ellas consume 2 comidas y una de las participantes solamente come una vez al día. Cuando se les pregunta acerca de su dieta, se establece que su base alimenticia está constituida básicamente por carnes, verduras y granos, estando ausentes las frutas, los lácteos en la mayoría de los casos, así como es frecuente que incluyan comidas de paquete, gaseosas y alimentos fritos de venta callejera.

Así describe María su alimentación diaria: "Chocolate y huevos, ese es el desayuno; me arreglo y salgo a las más o menos un cuarto para las 10 para el negocio y de ahí pues todo el día meseando y... ¿̇cuando llego a la casa? me acuesto (risa). Hay veces que no como, o sea, yo soy de muy mal comer, me la paso de pronto tomando gaseosas"

\section{Cambios en la actividad de prostitución debido al embarazo}

En la tabla 3 se describen de manera general los cambios que el embarazo ha generado con respecto a la actividad de prostitución de las mujeres.

La mayoría de las mujeres (9 de las 12) continúan ejerciendo la actividad de prostitución, aunque refieren que disminuye el número de clientes y que cambian algunas de sus prácticas durante la relación sexual; las tres restantes siguen relacionadas con el medio y en algunos casos actúan como "puente" entre los clientes y sus compañeras. Las razones que argumentan para disminuir la actividad tienen que ver con agotamiento físico y con el hecho de pensar que esto puede "incomodar o afectar al bebé", y con no querer someterse a las exigencias de algunos clientes. Además, mencionan que pierden interés por parte de los clientes debido a su apariencia. El hecho de reducir su actividad, tiene repercusiones negativas de tipo económico, según manifiestan las participantes que continúan en ella, básicamente para no verse tan afectadas en sus ingresos.

Juliana mencionó al respecto: "Ya es muy rara la vez que entre- se refiere a la actividad sexual- y con mi pareja también hemos decidido pararle un poquito por no lastimar el bebé, por muchas cosas"

Por su parte, Paulina comenta lo siguiente: “iAy! sí me da mucha flojera trabajar, no que pereza, no me gusta, o sea pensando en el bebé, que es incómodo también, para mí es incómodo irme a acostar con alguien que yo no siento absolutamente nada y que sé que hay una criaturita dentro de mí es feo, me da pereza trabajar, pero como te digo tengo

Tabla 3. Cambios en la actividad de prostitución debido al embarazo.

\section{Concepto}

Continúan con la actividad

Disminución de la actividad durante el embarazo

Disminución ingreso

\section{Respuesta}

Sí

No

Sí

No

Sí

No
Número de casos
3

10

2

10 
que hacerlo, hasta ahorita por ahí hasta el 22 de diciembre que pienso trabajar(...) ya se ve el mondongo, ya esto es un mondongo (se ríe y se toca el abdomen) si ya se ve el estómago entonces las cosas ya no son iguales, me siento mal porque ya llegan y de pronto ya no está esa niña sexy, ya se siente uno como más.. ¿si me entiendes? como más aisladito, como más rechazado, por más que sea no van a querer barrigoncitas, pero, como todo, todos tenemos nuestros días, hay días buenos otros días que uno se las pasa en blanco o sin bajar bandera como le dicen algunas(. . .). A nosotras nos toca hacer show, las que quieran, se paga 15.000 pesos y de mesa, sentándose con los clientes para motivar el consumo de licor, \$20.000. Digamos yo ahorita hago sólo de mesa o en la habitación digo que no, que en privado".

\section{Redes sociales}

La soledad es común en el diario vivir de las mujeres de este grupo. Sus escasas redes sociales, en la mayoría de los casos, están representadas por sus compañeras, por personas vecinas, y en contadas ocasiones por sus parejas, como es el caso de Juliana: "Él es el que me apoya, el que me ayuda, pero así que yo tenga problemas aquí con lo que estoy haciendo, me toca es solita, o sea, cuento con los jefes y con los compañeros, pero de resto no"

Así relata, por su parte, su situación Jenny, quien afirma que cuenta con el apoyo de personas que la rodean en su medio: "Como yo vivo con travestis, la dueña de la casa es una travesti; ella ya está viejita y ella me quiere mucho porque yo a ella le colaboro cuando estoy aliviada, entonces: 'vea, Úrsula, estoy embarazada y tengo un mes', 'ay, mija, ¿cómo así? pero tranquila, qué se le va hacer...' y a otra amiguita, que ella es de Pereira, también le compartí y ella me estuvo colaborando, pero le tocó irse a viajar"

\section{Percepción de los servicios de salud}

Del grupo de 12 mujeres, 11 manifiestan estar satisfechas con los servicios de salud que han recibido, a pesar de que varias actualmente no asisten a controles y tres no están afiliadas ni vinculadas al sistema. Quien afirma no estar totalmente satisfecha, es una mujer que está vinculada al régimen contributivo, quien observa lentitud en la prestación del servicio. Varias de las participantes afirman que ocultan a los profesionales de la salud el hecho de estar en situación de prostitución para no verse discriminadas. Así lo narra Sara: "En la EPS bien, pues como persona normal, ya que no saben lo que hago. Porque hay mucha gente que rechaza eso, pues, por miedo a ese rechazo es que no, muchas veces no cuento que yo trabajo en eso"

\section{Proyecto de vida}

Ante la pregunta de cómo se imaginan su vida en diez años, se evidencia en las participantes el deseo de tener una vida estable, en compañía de sus hijos, de "trabajar dignamente" y de contar con los ingresos necesarios para tener una calidad de vida. Llama la atención que la mayoría no incluyen a su pareja en sus planes futuros o dudan de la posibilidad de compartir con alguien. De esta manera lo narra Paulina: "No todavía no he pensado hasta allá, en 10 años he pensando en mis planes de estar junto a mis hijos, no trabajar en esto, porque yo pensaba trabajar por ahí dos años, yo había pensado en salirme, pero ahorita me toca pagar todas las cosas y mirar más adelante cuando tenga mi bebé, la señora en donde vivo me decía que muy bueno que ese muchacho me iba a responder, que de pronto podíamos formar un hogar, bueno... aunque yo tampoco aspiro a tener marido..."

\section{DISCUSIÓN}

Algunos factores sociodemográficos han sido reconocidos como de riesgo para la gestación. La pobreza, los bajos niveles educativos y edades menores de 17 años y mayores de 35 años, hacen parte de los aspectos que, de manera más frecuente, pueden ser predictores de complicaciones maternas. ${ }^{13,10}$ En este grupo, como se ha establecido, dos mujeres están en edades de alto riesgo, siendo una de ellas menor de 17 años y otra mayor de 35.

Es bien sabido que los factores de riesgo asociados a las prácticas sexuales, la utilización incorrecta de métodos anticonceptivos, el consumo de alcohol y sustancias psicoactivas durante la maternidad (desde la gestación), así como los hábitos precarios de 
higiene genital, entre otros, conllevan al deterioro precoz de la salud de la mujer y de la población infantil. ${ }^{11}$ En este grupo humano se evidencian riesgos asociados a la falta de protección sexual.

La anticoncepción también constituye una problemática de serias dimensiones, ya que el no uso de anticonceptivos se estableció en siete de las 12 mujeres. En dos de los casos, las mujeres atribuyen su embarazo a la falla del condón, quizás por uso inadecuado, lo que se corroboró con sus relatos en los talleres realizados con las mujeres al inicio del proceso, varias se refirieron al uso del "doble preservativo" y de lubricantes que no son a base de agua, situaciones conocidas como factor de riesgo para que se rompa el condón. ${ }^{7}$ Ambas situaciones pueden ser abordadas por el personal de salud al explicar adecuadamente el concepto de doble protección (no doble método) para protegerse de ITS y gestación simultáneamente, así como explicar cuáles lubricantes a base de agua pueden utilizarse.

El consumo de alcohol, SPA y tranquilizantes es cotidiano en las mujeres que participaron en este estudio.

De acuerdo con la OPS (2005), "las limitaciones dentro de las cuales las mujeres viven su sexualidad y su acceso a programas preventivos, condicionan en gran medida sus comportamientos de autocuidado”. Su salud sexual y reproductiva, así como sus precarias prácticas de autocuidado, constituyen una de las mayores problemáticas que generan vulnerabilidad en este grupo de mujeres. El hecho de realizar actividades dentro del campo de la prostitución, las predispone a ITS/VIH/SIDA. ${ }^{14}$

La violencia es otro antecedente del riesgo materno y estas mujeres se desenvuelven en ambientes donde el maltrato es frecuente. ${ }^{15}$

Con relación a su estado de salud, si bien ocho de las doce mujeres afirman tener un buen estado de salud, una cuarta parte de ellas reporta complicaciones debidas a hipertensión asociada al embarazo, aborto fallido y sífilis. Los abortos repetidos y la multiparidad son aspectos que se asocian de igual manera con los riesgos maternos; ${ }^{11,13}$ en varios de los casos se presenta historia de ambos determinantes. El hecho de que un embarazo sea no planeado o no deseado se considera un factor de riesgo. ${ }^{15}$ Los abortos inseguros son frecuentes en este grupo en el que además las mujeres muestran, de manera frecuente, más de tres embarazos, a pesar de ser la mayoría muy jóvenes.

La no asistencia a control prenatal en una tercera parte de las entrevistadas, así como la poca participación en los cursos de preparación para la maternidad y la paternidad responsables, son factores de riesgo identificados en este grupo.

La situación en que se encuentran inmersas estas mujeres refleja una condición de género marcada por la inequidad y la violencia. Sus historias familiares, la edad en la cual iniciaron su vida sexual, los antecedentes de abuso sexual, el tipo de relaciones afectivas que suelen tener; informan acerca de vidas inestables, marcadas por el maltrato, el desamor y la soledad. En muchos casos tienen hijos que no terminan educando y es frecuente el embarazo no planeado o indeseado, así asuman con sumisión y como una deuda moral que contraen por la actividad que realizan, el proceso de gestación. Es notorio cómo las razones por las cuales disminuyen su actividad, más que estar relacionadas con su autocuidado, se relacionan con su construcción de lo moral, ya que sienten vergüenza y perciben que pueden "afectar al bebé" con el acto sexual.

La violencia de sus parejas, según sus relatos, es otro aspecto que atenta contra su salud. El embarazo en este grupo es, en cierta medida, una "amenaza" frente a su actividad, ya que, según ellas, se ven afectadas en el sentido económico. Desde su percepción, más que considerar su actividad un riesgo para su embarazo, su embarazo "pone en riesgo" su actividad.

Su concepto del deber ser femenino, que condiciona su noción de la maternidad y de lo que significa un hijo, y que las lleva a asumir el hecho como parte de lo que deben necesariamente vivir, sin que medien las decisiones; además de los riesgos 
en su salud y de la precariedad de su autocuidado, hablan de la necesidad de reconocer en este grupo humano y en sus hijos e hijas unas circunstancias de alto riesgo.

En un estudio realizado por Urrea y sus colaboradoras de la facultad de odontología de la Universidad El Bosque, en el año 2006, en el Centro Asistencial Ricaurte-San José de la localidad los Mártires, zona en la cual están ubicadas varias de las participantes en este trabajo, se llevó a cabo la revisión de 83 historias clínicas de mujeres en estado de embarazo, pertenecientes a los estratos 1 y 2, encontrando riesgos de salud oral en 77 casos. Otros riesgos destacados fueron aquellos asociados a la salud sexual y reproductiva; se hallaron, entre otros, 21 casos con antecedentes de aborto y 18 casos con diagnóstico de vaginosis. ${ }^{14}$

Si bien algunos hallazgos de este estudio coinciden con aquellos obtenidos en el estudio de Urrea y colaboradoras, con mujeres de la localidad los Mártires; las condiciones sociales y emocionales en este grupo parecen ser especialmente complejas y su actividad las lleva a mostrar mayor frecuencia de consumo de alcohol y SPA; a exponerse, por su actividad, a la noche y a la calle, con todo lo que ello conlleva y a verse mayormente solas y sin contar con quien las pueda apoyar.

Un estudio de la Universidad Externado de Colombia para el Departamento Administrativo de Bienestar Social, hoy Secretaría Distrital de Integración Social, realizado en 2002, sobre la explotación sexual a la infancia en Bogotá, encontró 9 niñas en condición de embarazo con edad promedio de 13 años. Los embarazos de estas niñas provenían, según los investigadores, de relaciones sexuales con explotadores que prefieren el sexo sin condón e incluso están dispuestos a pagar más dinero para lograrlo; y en otras ocasiones, del "marido", que por lo general es un hombre joven dedicado a la delincuencia, que les suele imponer el embarazo como parte de relaciones de carácter explotativo y a la vez protector, en las cuales impera la dominación masculina. ${ }^{15}$
Procesos de empoderamiento y de formación para el fortalecimiento de la autoestima y del autocuidado, que lleven a estas mujeres a conocer y a tener conciencia de su cuerpo, con énfasis en sus derechos, así como procesos de acompañamiento durante la gestación, que promuevan la asistencia a controles y las redes sociales de carácter institucional, pueden tener un impacto favorable en esta población.

La mayor limitación de este método de investigación, es que los hallazgos no se pueden generalizar o extender a todas las personas que ejercen la prostitución, dado que esta metodología centra el interés en algunos pocos sujetos de estudio, para poder conocer el contexto biopsicosocial en el que se desenvuelven.

\section{AGRADECIMIENTOS}

A la Dra. Isabel Pachón por sus aportes y a la Subdirección de Integración de los Mártires por abrirnos sus puertas y permitir la realización de este estudio. A todas las mujeres entrevistadas, asistentes a capacitación en la Subdirección de Integración de los Mártires y a las mujeres que participaron voluntariamente por su valiosa colaboración.

\section{REFERENCIAS}

1. Organización Mundial de la Salud. Informe sobre la salud en el mundo, 2005. Visitado en 2008 May 6. Disponible en: http://www.who. int/whr/2005/overview_es.pdf

2. Lafaurie MM. La prostitución en escena. Departamento Administrativo de Bienestar Social; 2002.

3. Secretaría Distrital de Salud de Bogotá, D.C. Política Sexualidad sin Indiferencia. 2006.

4. United Nations Population Fund (UNFPA) Annual Report; 2003.

5. Norma técnica para la detección temprana de las alteraciones del embarazo. Resolución número 00412, 2000. Ministerio de la Protección Social de Colombia.

6. Sistema Nacional de Vigilancia en Salud Pública, SIVIGILA. Situación de las enfermedades de 
transmisión sexual. Colombia. 14 al 20 de junio de 2003.

7. Gómez PI. Autonomía de los cuerpos. Anticoncepción una herramienta. Bogotá: Publimpacto; 2007.

8. Ministerio de la Protección Social. Dirección General de Salud Pública. Política Nacional de Salud Sexual y Reproductiva. Bogotá D.C.; 2003.

9. Thomas Muhr Atlas Ti software. Technical University of Berlin. 2007.

10. UNICEF. Djibouti. Resumen Oficial del Informe sobre el Estado Mundial de la Infancia; 2006.

11. Ministerio de la Protección Social. Situación de salud en Colombia: Indicadores Básicos. INS, OPS; 2003.

12. Organización Panamericana de la Salud. Intervenir sobre los determinantes sociales y mejorar la calidad de la atención del servicio de salud. La clave para que Colombia logre las Metas de Desarrollo. Bogotá D.C.; 2005.
13. Vladislavovna D, Pámanes-González V, Billings D, Torres-Arreola L. Violencia de pareja en mujeres embarazadas en la Ciudad de México, Rev. Saúde Pública 2007;41:582-90. Visitado en 2008 Abr 14. Disponible en: http://www.scielosp. org/scielo.php?pid $=$ S0034-8910200700040001 $2 \&$ script $=$ sci_arttext $\&$ tlng $=$ en

14. Urrea F, Lafaurie MM, Sandoval MA, Contreras $\mathrm{N}$, Hurtado Y, Páez A. Una mirada de género a los riesgos de salud de la mujer en situación de embarazo. Ponencia en XVI Congreso Institucional de Investigación Universidad El Bosque, 3 de septiembre de 2008 .

15. Aponte M, García CI. Explotación sexual infantil en Bogotá. Departamento Administrativo de Bienestar Social - Universidad Externado de Colombia, Bogotá; 2002. 